Original Paper http://ajol.info/index.php/ijbcs nttp://indexmedicus.afro.who.int

\title{
Farine de sevrage commerciale ANAGOBAKA: quels risques pathologiques dans le régime du rat en croissance?
}

\author{
Egnon K.V. KOUAKOU ${ }^{1 *}$, Kouamé G. M. BOUAFOU ${ }^{2}$, Alassane MEITE ${ }^{1}$, \\ K. Gustave KOUAME ${ }^{1}$ et Séraphin KATI- COULIBALY ${ }^{1}$ \\ ${ }^{1}$ Laboratoire de Nutrition et Pharmacologie, UFR Biosciences, \\ Université Félix Houphouët-Boigny 22 B.P. 582 Abidjan 22, Côte d'Ivoire. \\ ${ }^{2}$ Section Sciences de la Vie et de la Terre, Ecole Normale Supérieure d'Abidjan08 B.P. 10 Abidjan 08, \\ Côte d'Ivoire. \\ *Auteur correspondant, E- mail : kouakouegnonvivien@yahoo.fr, Tel : (+225) 084993 34/(225) 40897897
}

\section{RESUME}

Trois groupes de dix rats en croissance sont nourris durant 15 jours avec trois régimes alimentaires dont deux farines de sevrage du commerce qui sont l'Anagobaka 2,04\% de protéines et le Cerelac Blé avec 14,94\% de protéines. Un régime témoin constitué de farine de poisson fixé à $14,94 \%$ de protéines. Le dosage des paramètres plasmatiques et la biométrie des reins, cœurs, poumons, iléons et des foies sont effectués chez cinq rats de chaque lot en fin d'expérience. Les valeurs obtenues avec l'urée $(0,18 \pm 0,05 \mathrm{~g} / \mathrm{l})$, la glycémie $(1,04 \pm$ $0,32 \mathrm{~g} / \mathrm{l})$, et la créatinine $(0,47 \pm 0,06 \mathrm{~g} / \mathrm{l})$ chez les rats consommant l'Anagobaka sont inférieures à celles des rats soumis aux régimes Cerelac Blé (urée $0,22 \pm 0,01 \mathrm{~g} / \mathrm{l}$; glycémie 2,94 $\pm 0,71 \mathrm{~g} / \mathrm{l}$; créatinine 0,72 $\pm 0,32 \mathrm{~g} / \mathrm{l}$ ) et témoin (urée 0,24 $\pm 0,02 \mathrm{~g} / \mathrm{l}$; glycémie 2,91 $\pm 0,04 \mathrm{~g} / \mathrm{l}$; créatinine $0,72 \pm 0,08 \mathrm{~g} / \mathrm{l}$ ). La biométrie des organes a révélé chez les rats sous régime Anagobaka la diminution de 34,70\% du poids de l'iléon et l'augmentation de $16,92 \%$ des reins, $21,20 \%$ du poids du foie et de $52,17 \%$ du poids du cœur en comparaison aux sujets témoins. Ces résultats pourraient présager une pathologie ou une perturbation du métabolisme nutritionnel de ces organes chez les rats soumis au régime Anagobaka.

(C) 2016 International Formulae Group. All rights reserved.

Mots clés : Farine, sevrage, biométrie, métabolisme.

\section{Commercial weaning flour ANAGOBAKA: what pathological risks in the growing rat diet?}

\begin{abstract}
Three groups of ten young rats are fed during 15 days with three diets among which two weaning meals namely Anagobaka 2,04\% of proteins and wheat Cerelac 14,904\% of proteins. The other is an experimental diet made of fish flour with $14,904 \%$ of proteins. At the end of the experiment, a dosage of biochemical plasma parameters and the biometry of kidneys, hearts, lungs, ileums and livers are realized on "all the rats". The results obtained with after urea $(0,18 \pm 0,05 \mathrm{~g} / \mathrm{l})$, blood glucose analysis $(1,04 \pm 0,32 \mathrm{~g} / \mathrm{l})$, and the creatinine $(0,47 \pm 0,06 \mathrm{~g} / \mathrm{l})$ on rats fed with Anagobaka are inferior to those of rats fed with Wheat Cerelac (urea $0,22 \pm$
\end{abstract}


$0,01$; blood glucose analysis $2,94 \pm 0,71 \mathrm{~g} / \mathrm{l}$; creatinine $0,72 \pm 0,32 \mathrm{~g} / \mathrm{l})$ and to those of the first experiment rats (urea $0,24 \pm 0,02 \mathrm{~g} / \mathrm{l}$; blood glucose analysis $2,91 \pm 0,04$; creatinine $0,72 \pm 0,08$ ), The dosage of biochemical plasma parameters could reveal some physiological pathologies and handicap. Yet, the organs biometry for rats submitted to Anagobaka diet has revealed the diminution of 34,70\% from the weight of ileum, and the raise in weight of $16,92 \%$ of the kidneys, $21,20 \%$ in weight of liver and $52,17 \%$ in the in weight of heart compared to those of the first experiment rats. These results could foretell a pathology or a disturbance of the nutrition metabolism of these organs.

(C) 2016 International Formulae Group. All rights reserved.

Keywords: Flour, weaning, biometrics, metabolism.

\section{INTRODUCTION}

Le sevrage constitue une agression nutritionnelle pour le nourrisson habituellement nourri exclusivement au lait maternel. Pendant cette période de la vie du nourrisson et de l'enfant, apparaissent les divers signes de malnutrition protéinoénergétique (Bouwer et al., 2000 ; Kafuti et al., 2015). Durant cette période dite de "sevrage", le nourrisson a besoin de farine de sevrage qui puisse lui fournir suffisamment d'énergie, de protéines, de vitamines, de minéraux et d'oligoéléments (Dupont, 2005). La qualité des farines infantiles utilisées pendant cette période est de ce fait d'une grande importance. En Afrique, pendant le sevrage, les mères nourrissent généralement leurs enfants avec des bouillies préparées à partir de farines simples ou composées provenant de céréales, de tubercules qui sont des aliments riches en glucides et pauvres en protéines (Zannou et al., 2011 ; Kouassi et al., 2015). Ces aliments sont incapables de couvrir tous les besoins nutritionnels de l'enfant (Sawadogo et al., 2003 ; Elenga et al., 2009) et l'Anagobaka ferait partie de cette catégorie de farines de sevrage.

Les paramètres biochimiques plasmatiques et biométriques des organes sont des techniques d'exploration de routine des organes du métabolisme nutritionnel (Bouafou et al., 2011). Cette étude vise donc à déceler d'éventuelles pathologies dans le foie, le cœur, l'iléon, les poumons et les reins des rats en croissance, nourris à l'Anagobaka, par le dosage de leurs paramètres biochimiques plasmatiques et par la biométrie de leurs organes.

\section{MATERIEL ET METHODES \\ Matériel \\ Animaux et logement}

Les animaux ont été des rats de souche Wistar en croissance (55 g à $57 \mathrm{~g}$ ) issus du Laboratoire de Nutrition et Pharmacologie de l'Université Félix Houphouët-Boigny, Abidjan (Côte d'Ivoire). Les rats ont été logés dans des cages à métabolisme individuelles à fonds grillagés qui permettent de retenir les fèces en amont et de recueillir en aval les urines, qui se déversent dans des bocaux par un entonnoir fixe. Les cages munies de râteliers et de biberons pour alimenter et abreuver les animaux.

\section{Matériel alimentaire}

Trois farines de sevrage infantiles ont été proposées aux rats en croissance sous forme de purée dont deux issues du commerce (Anagobaka et Cerelac Blé de Nestlé) et un régime témoin. Le taux de protéine du régime témoin est fixé à $14,94 \mathrm{~g} / 100 \mathrm{MS}$ avec un niveau énergétique égal à $4200 \mathrm{kcal} / \mathrm{kg}$ de matières sèches. Les proportions de glucides et de lipides des régimes sont obtenues par calcul. L'amidon de maïs (Maïzena) a été la principale source de glucides du régime témoin. L'huile de maïs (Lesieur) a été utilisée dans la formulation du régime témoin pour son apport en lipide et en acides gras essentiels. La farine de poisson représente la source de protéine du régime témoin. Le mélange vitaminé et minéral a été préparé 
selon les indications de Pawlak et al. (1968) (Tableau 1).

\section{Méthodes}

Conditions expérimentales et constitution des lots de rats

La salle d'expérience avait une température de $25{ }^{\circ} \mathrm{C}$, avec un degré d'hygrométrie compris entre 70 et $80 \%$. Trois lots de dix rats en croissance ont été soumis chacun aux régimes alimentaires.

\section{Conduite de l'expérience}

L'expérimentation animale s'est faite selon la méthode d'Adrian et al. (1991). Les régimes sont distribués ad libitum une fois par jour (de 6 h 30 min à 7 h $30 \mathrm{~min}$ ) sous forme de purée pendant 15 jours. L'eau est servie à volonté et renouvelée tous les trois jours.

Dosage des paramètres biochimiques plasmatiques et biométrie des organes

En fin d'expérimentation animale, cinq jeunes rats de chacun des régimes sont sacrifiés sous anesthésie à l'éthyle uréthane à $20 \%$, le matin entre 11 heures et 15 heures sans avoir subi de jeûne (Pawlak et al., 1968). Le sang est prélevé au niveau de la carotide dans des tubes numérotés (Pawlak et al., 1968). Ces échantillons de sang sont aussitôt transportés au Laboratoire de Biochimie médicale du Centre de Santé Communautaire Angré Ninatoullah, dans une glacière pour analyses.

Les dosages se sont conformés aux prescriptions des réactifs des kits du groupe espagnol SPINREACT S.A. (Digeon et al., 1975; Fawcett et al., 1960; Young et al., 1975). La valeur de chacun des paramètres biochimiques plasmatiques (en $\mathrm{mg} / \mathrm{dl}$ ) a été déterminée à partir de la relation suivante :

$\mathrm{P}=$ [D.O. écht/D.O. standard] $\mathrm{x}$ concentrations standard, où $\mathrm{P}$ est le paramètre biochimique plasmatique à doser, la D.O. écht (densité optique de l'échantillon) à $610 \mathrm{~nm}$ et la concentration standard est de $200 \mathrm{mg} / \mathrm{dl}$.

\section{Prélèvement et pesée d'organes}

Les reins, le cœur, les poumons, l'iléon et les foies ont été prélevés rapidement sur les jeunes rats sacrifiés. Ils sont déshumidifiés sur du papier filtre et pesés.

\section{Analyse statistique}

Les résultats sont exprimés sous moyenne \pm écart-type. Les comparaisons des valeurs des paramètres biochimiques plasmatiques et biométriques des organes ont été effectuées à partir du logiciel STATISTICA, version 6.0, par le test de Newman Keuls à $5 \%$ a été utilisée à cet effet.

\section{RESULTATS \\ Etude des paramètres biochimiques plasmatiques}

Les valeurs obtenues avec l'urée $(0,18$ $\pm 0,05 \mathrm{~g} / \mathrm{l})$, le rapport $\mathrm{Ca} / \mathrm{P}(0,46 \pm 0,03)$, la glycémie $(1,04 \pm 0,32 \mathrm{~g} / \mathrm{l})$, les triglycérides $(0,32 \pm 0,32 \mathrm{~g} / \mathrm{l})$ et la créatinine $(0,47 \pm 0,06$ $\mathrm{g} / \mathrm{l})$ chez les rats consommant l'Anagobaka sont inférieures à celles des rats soumis aux régimes Cerelac Blé (urée 0,22 $\pm 0,01 \mathrm{~g} / \mathrm{l}$; triglycérides $0,52 \pm 0,13 \mathrm{~g} / \mathrm{l}$; créatinine $0,72 \pm$ $0,32 \mathrm{~g} / \mathrm{l} ;$ rapport $\mathrm{Ca} / \mathrm{P} 0.94 \pm 0,11)$ et témoin (urée $0,24 \pm 0,02 \mathrm{~g} / \mathrm{l}$; triglycérides $0,60 \pm 0,18$ $\mathrm{g} / \mathrm{l}$; créatine $0,72 \pm 0,08 \mathrm{~g} / \mathrm{l}$; rapport $\mathrm{Ca} / \mathrm{P} 0,91$ $\pm 0,07$ ) ( Tableau 2). Au plan statistique, aucune différence significative n'est observée entre les jeunes rats des lots témoins et Cerelac Blé (p). Cependant, il existe une différence significative entre le régime Anagobaka et ceux du témoin et du Cerelac Blé $(\mathrm{p}<0,05)$.

\section{Biométrie des organes}

La biométrie des organes a décelé la diminution de $34,70 \%$ du poids de l'iléon, et l'augmentation de 16,92\% des reins, 21,20\% du poids du foie et de $52,17 \%$ du poids du cœur des rats sous régime Anagobaka. Les organes des rats soumis au régime Anagobaka sont significativement différents aux organes de ceux nourris aux Cerelac Blé et témoin (Tableau 3). 
Tableau 1 : Composition chimique des trois régimes alimentaires

\begin{tabular}{lccc}
\hline & Anagobaka & Cerelac Blé & Témoin \\
\hline Protéines g/100 MS & $2,04 \pm 0,03^{\mathrm{a}}$ & $14,94 \pm 0,16^{\mathrm{b}}$ & $14,94 \pm 0,9^{\mathrm{b}}$ \\
Glucides g/100 MS & $82,1 \pm 0,94^{\mathrm{a}}$ & $66,6 \pm 0,12^{\mathrm{b}}$ & $72,2 \pm 0,28^{\mathrm{b}}$ \\
Matière grasse g/100 MS & $6,04 \pm 0,11^{\mathrm{a}}$ & $12,0 \pm 0,8^{\mathrm{b}}$ & $8,8 \pm 0,06^{\mathrm{b}}$ \\
Valeur énergétique g/100 MS & $390,32^{\mathrm{a}}$ & $434,01^{\mathrm{b}}$ & $427,76^{\mathrm{b}}$ \\
Fer mg/100 MS & $3,08 \pm 0,05^{\mathrm{a}}$ & $7,6 \pm 0,27^{\mathrm{b}}$ & $6,25 \pm 0,02^{\mathrm{b}}$ \\
Calcium g/100 MS & $97,34 \pm 0,64^{\mathrm{a}}$ & $430 \pm 0,94^{\mathrm{b}}$ & $402 \pm 0,05^{\mathrm{b}}$ \\
Zinc g/100 MS & $1,35 \pm 0,04^{\mathrm{b}}$ & $1,8 \pm 0,00^{\mathrm{b}}$ & $2,35 \pm 0,23^{\mathrm{b}}$ \\
\hline \multicolumn{2}{l}{ Chaque valeur est la moyenne suivie de l'écart type de trois essais ; $^{\mathrm{a}, \mathrm{b}}:$ :il n'y a aucune différence } \\
significative entre 2 valeurs de la même colonne surmontée par la lettre.
\end{tabular}

Tableau 2: Valeurs des paramètres plasmatiques $(\mathrm{g} / \mathrm{l})$ des rats soumis aux différents régimes alimentaires

\begin{tabular}{|c|c|c|c|c|c|}
\hline & Glycémie & Triglycéride & Cholestérol & Urée & Créatinine \\
\hline Anagobaka & $1,04 \pm 0,32^{\mathrm{e}}$ & $0,32 \pm 0,02^{\mathrm{e}}$ & $0,47 \pm 0,05^{\mathrm{e}}$ & $0,18 \pm 0,05^{\mathrm{e}}$ & $0,47 \pm 0,06^{\mathrm{e}}$ \\
\hline Cerelac Blé & $2,94 \pm 0,73^{\mathrm{d}}$ & $0,52 \pm 0,13^{\mathrm{d}}$ & $0,78 \pm 0,03^{\mathrm{d}}$ & $0,22 \pm 0,01^{\mathrm{d}}$ & $0,72 \pm 0,05^{\mathrm{d}}$ \\
\hline Témoin & $2,91 \pm 0,04^{\mathrm{d}}$ & $0,60 \pm 0,18^{\mathrm{d}}$ & $0,73 \pm 0,06^{\mathrm{d}}$ & $0,24 \pm 0,02^{\mathrm{d}}$ & $0,72 \pm 0,08$ \\
\hline
\end{tabular}

Tableau 3: Résultats de la biométrie des organes.

\begin{tabular}{llll}
\hline & Anagobaka & Cerelac Blé & Témoin \\
\hline \%Poids (2) reins/poids corporel & $0,87 \pm 0,06^{\mathrm{f}}$ & $0,61 \pm 0,15^{\mathrm{g}}$ & $0,65 \pm 0,03^{\mathrm{g}}$ \\
\%Poids foie/poids corporel & $3,83 \pm 0,57^{\mathrm{f}}$ & $3,33 \pm 0,56^{\mathrm{g}}$ & $3,16 \pm 0,38^{\mathrm{g}}$ \\
\%Poids cœur /poids corporel & $0,70 \pm 0,57^{\mathrm{f}}$ & $0,42 \pm 0,04^{\mathrm{g}}$ & $0,46 \pm 0,04^{\mathrm{g}}$ \\
\%Poids iléon/poids corporel & $0,45 \pm 0,03^{\mathrm{f}}$ & $0,71 \pm 0,320^{\mathrm{g}}$ & $0,69 \pm 0,08^{\mathrm{g}}$ \\
\%Poids poumon/poids corporel & $0,79 \pm 0,78^{\mathrm{f}}$ & $0,83 \pm 0,07^{\mathrm{g}}$ & $0,81 \pm 0,02^{\mathrm{g}}$ \\
\hline Chaque valeur est la moyenne suivie de l'écart type de cinq rats. \\
f,g g:aucune différence significative entre 2 valeurs de la même ligne surmontée par la lettre.
\end{tabular}

\section{DISCUSSION}

L'analyse des paramètres biochimiques plasmatiques et la biométrie des organes des rats ont permis d'évaluer les risques liés à la consommation des différents régimes (Anagobaka, témoin, Cerelac Blé). L'analyse des paramètres biochimiques plasmatiques et la biométrie des organes ont montré une différence significative entre le régime Anagobaka et les autres régimes (témoin, Cerelac Blé). Cependant, les valeurs plasmatiques sanguines et la biométrie des organes du régime témoin et le Cerelac Blé n'ont montré aucune différence significative.

Les jeunes rats ayant ingéré l'Anagobaka, ont la plus faible glycémie $(1,04 \pm 0,32 \mathrm{~g} / \mathrm{l})$ en comparaison avec celles des jeunes sujets témoins $(2,91 \pm 0,74 \mathrm{~g} / \mathrm{l})$ et Cerelac Blé $(2,94 \pm 0,73 \mathrm{~g} / \mathrm{l})$. La chute de la glycémie est synonyme d'hypoglycémie chez les rats nourris à l'Anagobaka. Elle s'expliquerait certainement par l'insuffisance de l'apport énergétique global (Feillet, 2000). Pour couvrir leurs besoins, ces rats utilisent 
toutes les réserves glucidiques. Ce qui se répercute également sur les taux plasmatiques en lien avec l'utilisation du glucose issu de l'aliment.

La totalité des organes sont consommateurs de glucose afin d'assurer leur survie et leurs fonctions. Ce qui pourrait expliquer aussi l'amaigrissement des sujets soumis à ce régime malgré la forte consommation. Toutefois, la glycémie doit être maintenue dans des limites strictes afin de ne pas mettre l'intégrité de l'organisme en péril. Cette hypoglycémie observée chez les rats, malgré la forte consommation de l'Anagobaka s'expliquerait par un état de malnutrition protéino-énergétique dû à la carence en protéine et en énergie de ce dernier entraînant ainsi le Kwashiorkor.

La faible urémie $(0,18 \pm 0,05 \mathrm{~g} / \mathrm{l})$ observée chez les rats nourris à l'Anagobaka par rapport au régime témoin $(0,52 \pm 0,13 \mathrm{~g} / \mathrm{l})$ et Cérelac Blé $(0,71 \pm 0,05 \mathrm{~g} / \mathrm{l})$ pourrait attester une anomalie dans la physiologie rénale de ces animaux. L'urée provient de la destruction des protéines. Son excrétion se fait principalement par les reins et son taux reflète le fonctionnement global des reins (Maurizi et Zaoui, 2005). Ainsi, certains facteurs tels que la malnutrition protéinoénergétique et le dysfonctionnement hépatique peuvent aussi diminuer l'urémie (Lagrange, 2010). Ces anomalies pourraient confirmer les signes de kwashiorkor observés chez les animaux nourris à l'Anagobaka avec ses effets délétères sur les organes vitaux notamment les reins.

La créatinémie est, tout comme l'urémie, utilisée comme marqueur indirect de la fonction rénale (Séronie et al., 2004). Une faible créatinémie $(0,47 \pm 0,06 \mathrm{~g} / \mathrm{l}$ comparé au témoin et au Cerelac Blé respectivement 0,60 $\pm 18 \mathrm{~g} / \mathrm{l} ; 0,72 \pm 0,08 \mathrm{~g} / \mathrm{l}$ est également observée.

Cette faible production de créatinine chez les rats nourris à l'Anagobaka pourrait attester une anomalie dans la physiologie rénale de ces animaux (Coffi et al., 2014). En effet, la créatinine est formée dans le muscle à partir d'une dégradation non enzymatique de la créatine et est éliminé uniquement par les reins à travers le glomérule (Pierre et al., 2010). Le taux sanguin de la créatinine est un meilleur indicateur de la fonction rénale.

Ainsi, de faibles productions de créatinine et de l'urée ont montré une hypertrophie et des lésions de fibroses interstitielles importantes des reins chez les chiens (Velanovsky, 2003), mais aussi chez les rats atteints de malnutrition (Denise et Hervé, 2001).

En somme, ces faibles valeurs de l'urée et surtout de la créatinine observées chez les rats nourris à l'Anagobaka pourraient justifier ces dysfonctionnements rénaux. En effet, la biométrie des reins a montré une augmentation de $16,92 \%$ du poids corporel des reins des animaux nourris avec l'Anagobaka, contrairement aux régimes témoins et Cerelac Blé qui n'ont révélé aucune anomalie. Des observations similaires ont été rapportées par Zannou et al. (2011) qui indiquent que des rats nourris avec des régimes contenant des mélanges des farines de manioc avec du soja ont des reins ayant des poids supérieurs à ceux des rats nourris avec le régime caséine. Le rein est un organe extrêmement important, non pas par sa taille, mais par son fonctionnement. La structure du rein en fait un organe extrêmement efficace mais aussi extrêmement sensible aux différentes substances pouvant entraîner des anomalies. Ces valeurs anormales de la créatinine et de l'urée pourraient justifier l'hypertrophie rénale observée chez les animaux soumis au régime Anagobaka.

De même, de faibles valeurs de cholestérol $(0,47 \pm 0,05 \mathrm{~g} / \mathrm{l})$ et triglycéride $(0,32 \pm 0,02 \mathrm{~g} / \mathrm{l})$ ont été enregistrées chez les rats nourris à l'Anagobaka comparé respectivement au régime témoin $(0,73 \pm 0,06$ 
$\mathrm{g} / \mathrm{l})$ et $(0,60 \pm 0,18 \mathrm{~g} / \mathrm{l})$, et Cérelac Blé $(0,78 \pm$ $0,03 \mathrm{~g} / \mathrm{l})$ et $(0,52 \pm 0,13 \mathrm{~g} / \mathrm{l})$. Le cholestérol et les triglycérides sont produits dans des proportions assez importantes dans le foie pour approvisionner l'ensemble de l'organisme (Thibaut, 2012).

La faible production de cholestérol associée à l'insuffisance de triglycéride chez les animaux nourris avec l'Anagobaka pourrait entraîner une hyperactivité conduisant à une déformation du foie. En effet, la biométrie des organes a relevé une augmentation de $21,20 \%$ du poids corporel du foie des rats nourris à l'Anagobaka. Ce qui pourrait être assimilé à une hépatomégalie. Une situation comparable a déjà été observée chez les rats nourris avec des régimes contenant des asticots séchés (Bouafou et al., 2007), mais aussi chez les enfants souffrant de kwashiorkor (Feuillet, 2000). Elle s'explique par l'accumulation des lipides notamment de triglycérides et de cholestérols dans le foie (Ahmed et al., 2009). Ce qui pourrait confirmer le fait que la farine de sevrage de commerce Anagobaka contiendrait des substances difficilement métabolisables, imposant donc à ces organes un surcroît d'activité (Bouafou et al., 2011 ; Dahoudam et al., 2013).

Il est, en effet, observé aussi, chez les rats nourris à l'Anagobaka, une déformation des organes indicateurs de l'état nutritionnel. Ainsi, l'iléon des rats nourris avec l'Anagobaka a subi une hypotrophie de $34,70 \%$ du poids corporel alors que le cœur a augmenté de $52,17 \%$ de son poids corporel.

$\mathrm{Au}$ regard de ce qui précède, la consommation de ce produit sur une longue durée provoquerait donc des troubles graves du métabolisme conduisant à des dysfonctionnements et la mort des consommateurs sous l'effet du Kwashiorkor.

\section{Conclusion}

L'exploration par le dosage des paramètres biochimiques plasmatiques et la biométrie des organes, tels que les reins, le cœur, le foie, les poumons et l'iléon des rats ayant consommé l'Anagobaka, la farine de poisson (témoin) et le Cerelac Blé a été faite. Les paramètres biochimiques plasmatiques des rats soumis à l'Anagobaka sont significativement différents de ceux des rats nourris avec la farine de poisson (témoin) et le Cerelac Blé. De même que les paramètres biochimiques plasmatiques, la biométrie des organes a décelé certaines variations, la diminution de $34,70 \%$ du poids de l'iléon, et l'augmentation de $16,92 \%$ des reins, $21,20 \%$ du poids du foie et de $52,17 \%$ du poids du cœur des rats sous régime Anagobaka.

Ces données pourraient suggérer que la farine de l'Anagobaka, couramment utilisée dans les cas de sevrage en Côte d'Ivoire, pourrait avoir des effets néfastes sur la santé des rats en croissance, et par conséquent chez les enfants qui en consomment. Ces organes pourraient donc souffrir d'une pathologie ou d'une perturbation de leur métabolisme nutritionnel. Par conséquent, d'autres études seraient nécessaires, notamment sur le plan histopathologique, pour confirmer ou infirmer ces présomptions d'anomalies.

\section{CONFLIT D'INTÉRÊT}

Les auteurs déclarent qu'il n'y a aucun conflit d'intérêt sur cet article.

\section{REFERENCES}

Adrian J, Rabache M, Fragne R. 1991. Techniques d'analyse nutritionnelle. In Principes de Techniques d'Analyse. Ed Lavoisier TEC \& DOC : Paris ; 451-478.

Ahmed T, Sabuktagin R, Alejandro C. 2009. Oedematous malnutrition. Indian $\mathrm{J}$ Med Res., 130: 651-654.

Bouafou K, Konan BA, Meite A, Kouame G, Kati-Coulibally S. 2011. Substitution de 
la farine de poisson par la farine d'asticots séchés dans le régime du rat en croissance : risques pathologiques ? Journal of Applied Biosciences, 48: 3279-3283.

Bouafou K, Kouamé K, Offoumou M. 2007. Bilan azotée chez le rat en croissance de la farine d'asticots séchés. Tropicultura, 25(2) : 70-74.

Bouwer I, Traore A, Trêche S. 2000. Activités $\mathrm{du}$ 2ème atelier international. Voies alimentaire d'amélioration des situations des situations nutritionnelles en Afrique de l'Ouest. 23-28 Novembre, Ouagadougou, Burkina Faso. P 745-773

Coffi T, Ahissou H, Gouthon P, Laleye A. 2014. Etude de l'activité antihypertensive d'une association de plantes (Schrankia leptocarpa, Garcinia kola et Ocimum americanum) chez le rat Wistar. Int. J. Biol. Chem. Sci., 8(6): 2685-2695.

Dahouda M, Adjolohoun S, Senou M, Toleba S, Abou M, Vidjannagni D, Kpodekon M, Youssao A. 2013. Effets des aliments contenant les folioles de Moringa oleifera Lam et des aliments commerciaux sur les performances de croissance des lapins (Oryctolagus cuniculus) et la qualité de la viande. Int. J. Biol. Chem. Sci., 7(5): 1838-1852.

Denise E, Hervé L. 2001. Insuffisance rénale chronique: importance de la nutrition. Encyclopédie de la nutrition clinique canine, 33p.

Dingeon B, Ferry J, Roulet A. 1975. Automatic assay of blood sugar by Trinder's method. Ann. Biol. Clin., 33: 313.

Dupont C. 2005. La diversification alimentaire, cahier. Nutr. Diet. 40: 137250.

Elenga M, Massamba J,.Kobawila C, Makosso G, Silou T. 2009 Evaluation et amélioration de la qualité nutritionnelle des pâtes et des bouillies de maïs fermenté au Congo. Int. J. Biol. Chem. Sci., 3(6): 1274-1285.

Fawcett J, Scoot J. 1960. A rapid and precise method for determination of urea. J. Clin. Path., 13: 156-159.

Feuillet F. 2000. Adaptation métabolique à la malnutrition: modèle des lipides, de la cobalamine, de la riboflavine et des acides organiques dans la malnutrition proteino-énergétique de l'enfant et dans l'anorexie mentale. Doctorat 3è cycle. Université de Montpellier 2, p 203-218

Gerbouin P. 1996. Comment améliorer les aliments de complément du jeune enfant? Centre International de l'Enfance. 75016 Paris. Tirés à part, dans «Cahiers Santé» p 229-235.

Golden M. 1991. The nature of nutritional deficiencies in relation to growth failure andpoverty. Acta Paediatr. Scand., 374: 95-110

Heymsfield S, Arteaga C, Manus C, Smith J, Moffitt S. 1983. Measurement of muscle mass in humans: validity of the 24-hour urinary creatinine method. Am J Clin Nutr., 37: 478-494.

Kafuti M, Fundu M, Sindic M. 2015. Composition en acides aminés et en acides gras d'un aliment de complément à base des ressources alimentaires locales : cas de la Vamine en R D Congo. Int. J. Biol. Chem. Sci., 9(3): 1624-1629.

Kouassi A, Agbo A, Dago G, Gbogouri G, Brou K, Dago G. 2015. Comparaison des caractéristiques nutritionnelles et rhéologiques des bouillies infantiles préparées par les techniques de germination et de fermentation. Int. J. Biol. Chem. Sci., 9(2): 944-953.

Lagrange M. 2010. Microangiopathies thrombotiques, une urgence diagnostique. Option/Bio., 21(446): 16-7.

Packa T, Massamba A, Babela M, Senga P. 2010. Effets du tabagisme et du niveau de dépendance nicotinique sur les 
métabolismes anaérobies des footballeurs. Int. J. Biol. Chem. Sci., 4(6): 2082-2092.

Pawlak M, Pion R. 1968. Influence de la supplémentassions des protéines de blé par les doses croissantes de lysine sur la teneur en acides aminés libres du sang et du muscle du rat en croissance. Ann. Biol. Bioch. Biophys., 7: 517-530.

Pierre D, Etienne C, Nicolas M, Krzesinski JM, Christophe M, Cristol J-P, Laurence P. 2010. Créatinine : d'hier à aujourd'hui. Ann Biol Clin., 68(5): 531543.

Seronie S, Vivien M, Galteau M, Carlier M, Hadj A. 2004. Dosage de la créatinémie en 2003 : état des lieux analytique et essai de standardisation de l'étalonnage. Annales de Biologie Clinique, 62: 165175.

Thibaut D. 2012. Communication interorganes dans le contrôle du métabolisme glucidique: mise en évidence de l'implication du monoxyde d'azote et de l'apeline dans l'hypothalamus. Doctorat de l'université de Toulouse, p. 237

Velanovsky A. 2003. Modèles expérimentaux d'insuffisance rénale chez le chien. Doctorat de l'université Paul-Sabatier de Toulouse, P. 80.

Young D, Pestaner L, Gibberman V. 1975. Effects of drugs on clinical laboratory tests. Clin. Chem., 21: 5.

Zannou T, Bouafou K, Kouame G, Konan A. 2011. Etude de la valeur nutritive de farines infantiles à base de manioc et de soja pour enfant en âge de sevrage. Bulletin de la Société Royale des Sciences de Liège, 80: 748-758. 